\title{
Congenital Malaria and Its Associated Factors at Issaka Gazobi Maternity of Niamey in Niger
}

\author{
I. Tahirou, ${ }^{1}$ M. O. Zara, $^{2}$ M. L. Moustapha, ${ }^{3,4}$ M. Kamayé, ${ }^{1,5}$ D. Mahamadou, ${ }^{4}$ A. Ibrahim, ${ }^{1}$ \\ M. Daou, ${ }^{1,3}$ A. Soumana, ${ }^{1}$ and M. L. Ibrahim ${ }^{6}{ }^{6}$ \\ ${ }^{1}$ Faculté de Science de la Santé de l'Université de Niamey, Niger \\ ${ }^{2}$ Maternité Issaka Gazobi de Niamey, Niger \\ ${ }^{3}$ Université Cheick Anta Diop de Dakar, Senegal \\ ${ }^{4}$ Université de Zinder, Niger \\ ${ }^{5}$ Hôpital National de Niamey, Niger \\ ${ }^{6}$ Centre de Recherche Médicale et Sanitaire de Niamey, Niger
}

Correspondence should be addressed to M. L. Ibrahim; lamine.cermes@gmail.com

Received 23 December 2019; Revised 25 August 2020; Accepted 6 October 2020; Published 19 October 2020

Academic Editor: Samuel Menahem

Copyright (c) 2020 I. Tahirou et al. This is an open access article distributed under the Creative Commons Attribution License, which permits unrestricted use, distribution, and reproduction in any medium, provided the original work is properly cited.

\begin{abstract}
Background. Congenital malaria is a serious and common infection in tropical Africa. It has multiple consequences on the newborn and the mother. Objective. The objective of this study is to calculate the prevalence of congenital malaria, describe its clinical signs, and analyze its associated factors. Methodology. It is a cross-sectional and prospective study, conducted at Issaka Gazobi Maternity of Niamey, from June 1 to November 30, 2017. The diagnosis was made by microscopy of a thick and thin blood smear of mother, newborn, and umbilical cord. Results. Two hundred and forty-nine (249) consecutive newborn/mother pairs were included. The prevalence of congenital malaria infection was $26.51 \%(66 / 249)$ with a parasite density of $101 \mathrm{P} / \mu \mathrm{l}$ (SD: 47.3; [80; 320]). The prevalence of congenital malaria disease was $14.06 \%$ (35/249) with a parasite density of $108 \mathrm{P} / \mu \mathrm{l}$ (SD: 32.6; [40; 200]. All patients were infected with Plasmodium falciparum. 43\% (18/35) of neonates had hyperthermia and did not have a sucking reflex, $8.5 \%$ (3/35) were anaemic, $11.42 \%$ (4/35) had convulsed, $20 \%(7 / 35)$ had a coma, and $45.71 \%(16 / 35)$ had a low birth weight. No deaths were recorded, and only the nonuse of bed nets was significantly associated with congenital malaria $(p=0.04)$. Conclusion. In Niger, one out of four newborns is infected with Plasmodium. Infection can progress to congenital malaria disease. The use of mosquito nets and intermittent preventive treatment would reduce the incidence of congenital malaria.
\end{abstract}

\section{Introduction}

Congenital malaria is the in utero transmission of Plasmodium from mother to child during childbirth [1]. Biologically, it is the detection of red blood cells parasitized by the same species of Plasmodium, in the mother and the newborn before the seventh day of life. This contamination of the foetus is explained by the fact that the parasite can cross the placental barrier [2], following a decrease in the immunity of the mother [3].

In malaria-endemic areas, congenital malaria is a major public health problem according to the World Health Organization [1]. In tropical Africa, there are 25 million pregnant women each year and 6 million with malaria [4]. 75,000 to
200,000 newborn deaths are due to placental infections [3]. Congenital malaria is the second mode of transmission of malaria after vector transmission. Infections of the placenta are very common in malaria-endemic countries, particularly in primiparous women [5]. About 20.2\% of pregnant women have placentas infected [3, 6] by Plasmodium falciparum especially. This infection is a major cause of anaemia in pregnant women $[7,8]$. In foetus, this placental sequestration of parasitized red blood cells alters the integrity of the placenta, reduces the transport of oxygen and nutrients, resulting in low birth weight [3]. This foetal hypotrophy can reach $170 \mathrm{~g}$ [6]. Newborns are twice likely to have low birth weight in the presence of placental infection [9]. Placental malaria can also cause premature birth [10] and even death of the 
mother and/or child. 5.7\% of the deaths in Africa were due to placental infections during pregnancy [9]. Children should normally be protected by passive transmission of maternal immunoglobulin's, foetal haemoglobin, and colostrum [4]. However, it happens that children are born infected with Plasmodium. This infection can progress to clinical malaria known as congenital malaria disease.

The clinical manifestations of congenital malaria aren't most often specific [11]. The main clinical manifestations are fever, anaemia, and splenomegaly [8]. Jaundice, vomiting, and diarrhoea are also reported [12]. Biologically, it is characterized by parasitaemia and thrombocytopenia [13]. Two major clinical forms of congenital malaria are defined: (1) Congenital malaria infection, which is a transitory parasitaemia of peripheral blood of a neonate or its cord, clinically asymptomatic, and which spontaneously disappears in 2-3 days; (2) congenital malaria disease, which results in persistent parasitaemia with clinical manifestations during the first seven days of life. It has a spontaneous evolution, often fatal. These two forms must to be distinguished from the neonatal malaria, which is due to a postnatal inoculation of the parasite by the bite of infected female anopheles.

Congenital malaria infection is not uncommon in subSaharan Africa, and its prevalence varies from one site to another depending on the intensity of transmission [14]. This prevalence ranges from $10.8 \%$ to $54.2 \%$ [8]. In Niger, congenital malaria is poorly documented. Only two articles were published on the subject in 2000 [15] and 2006 [16].

It is in this context that we conducted a cross-sectional, prospective study to calculate the prevalence of congenital malaria, describe its clinical signs, and analyze its associated factors at Issaka Gazobi Maternity of Niamey-Niger.

\section{Methodology}

2.1. Methodology. It is a cross-sectional and prospective study to calculate the prevalence of congenital malaria, describe its clinical manifestations, and identify risk factors.

2.2. Site and Period of the Study. The study was conducted at Issaka Gazobi Maternity of Niamey, which is the National Reference Center (CNR) for Reproductive Health (RH), in collaboration with the Malaria Unit of the Center for Medical and Health Research, (CERMES) of Niamey. This site is located in a mesoendemic malaria transmission zone. This transmission is seasonal. It lasts 3 to 4 months, from July to October. The main vectors of malaria are Anopheles gambiae and Anopheles coluzzi. Plasmodium falciparum is the main transmitted parasite [17]. The study was conducted for sixmonths, from June 1 to November 30, 2017.

2.3. Study Population. The study population consisted of 249 consecutive mother/newborn pairs.

2.3.1. Inclusion Criteria. Were included in this study all term neonates, hospitalized at Issaka Gazobi Maternity neonal department and their mothers. They are 0 to 7 days old.

2.3.2. Criteria of Non Inclusion. Were not included in this study, all newborns without a health record, those who received a blood transfusion, premature babies and those whose parents did not consent.

2.4. Sampling. We used the simple probabilistic method. The inclusion of newborns followed the pace of hospitalization. With an estimated prevalence of $20 \%$ congenital malaria, a $95 \%$ confidence interval, and an accuracy of $5 \%$, the sample size was 246 subjects.

2.5. Collection of Data. Data were collected from an individual clinical record to trace the history of each patient. The variables collected on mothers are the identifier, the age, the origin of the patient, the number of prenatal consultations, the notion of malaria or a notion of treatment of malaria during pregnancy, the observance of intermittent preventive treatment (IPT), the use of a long-lasting insecticide-treated net (LLIN), and a biological examination to evaluate the plasmodial infection.

The variables collected on the newborn were gestational age, birth weight, delivery route, newborn Apgar at birth, amniotic fluid colour, general condition of newborns (temperature, heart rate, respiratory rate), convulsive number, thick blood smear (TBS), thin smear (TS), blood count, Protein C Reactive (CRP), thick blood smear and thin smear cord blood, parasite density, plasmodial species, evolution of clinical signs, thick control after treatment, duration of hospitalization, and finally the way out of newborns.

2.6. Diagnosis of Congenital Malaria. Microscopy, which is the WHO reference method for diagnosing malaria, was used. Each recruited newborn received a thick blood test (TBT) and thin smear (TS) of peripheral blood and umbilical cord blood by layer. If he is positive at birth, a TBT and TS control were performed at 48 hours.

Each recruited mother had received a thick blood test and thin smear of peripheral blood from her fingertips.

2.6.1. Thick Drop and Thin Smear. The blades of each patient are identified with the number of his medical file. The preparation of thick blood test and thin smears was made in accordance with WHO protocol [18].

2.6.2. Staining of the Blades. The dried slides were stained with May Grunwald Giemsa diluted 1/10 for 15 minutes. They are then rinsed and left in the open to dry.

2.6.3. Reading Slides. The slides were read under an optical microscope at 100X magnification. The thick drop was used to search and count the parasite. The parasite density was calculated by counting the number of parasites in parallel with 200 leucocytes. The parasite density is determined by the following formula: $\mathrm{PD}=$ Number of parasite counted $\times 8000 / 40$. The thin smear was used to identify the plasmodial species.

2.6.4. Haematological et Biochemical Tests. All haematological and biochemical examinations were performed at Issaka Gazobi Maternity Biology Laboratory using an automatic device (Reference: MSLAB O7 Plus). The parameters measured are red blood cell count, white blood cell count, hematocrit, platelets, blood sugar, and CRP. 
2.7. Ethical Consideration. This study was authorized by the Faculty of Health Sciences of the University of Niamey as part of its doctoral dissertation work for 2017-2018. The anonymous nature of the questionnaire makes it possible to guarantee the confidentiality of the information collected. The informed consent of all mothers was obtained before their inclusion.

2.8. Ethical Consideration. An entry mask was created using Epi Info 7.0 software. All data has been entered and processed with this software. Chi-square Pearson and Fisher tests were used to compare percentages. A threshold of significance of $5 \%$ was retained.

\section{Results}

\subsection{Characteristics of the Population}

3.1.1. Characteristics of Mothers. Two hundred and fortynine (249) mothers were included in the study. The mean age of mothers was 27 years (SD: 6.2, [12; 42]). 92.77\% (240/249) of women came from the Urban Community of Niamey (UCN), and 7.23\% (9/249) came from rural areas. 96.79\% (241/249) had given birth to Issaka Gazobi Maternity, and $3.21 \%(8 / 249)$ had been referred.

3.1.2. Characteristics of Newborns. Two hundred and fortynine (249) newborns were also included in the study. The sex ratio of newborns was 0.9 . They were all born to terms. The age of newborns at inclusion varies from zero to seven days. Their average weight was $2754 \mathrm{~g}$ (DS: 588; [1050; 4600]). Their average size was $47.23 \mathrm{~cm}$ (DS: 3.31 ; [34; 54]). The characteristics of the population are summarised in Table 1.

\subsection{The Prevalence of Congenital Malaria}

3.2.1. Prevalence of Congenital Malaria Infestation. $26.51 \%$ (66/249) of newborns had a positive tick drop. The mean parasite density was $101 \mathrm{P} / \mu \mathrm{l}$ (DS: 47.3, [80; 320]). All infections (100\%) were Plasmodium falciparum.

3.2.2. Prevalence of Congenital Malaria Disease. Forty-eight hours after the first biological examination, the thick blood drop remained positive in $14.06 \%$ (35/249) of neonates with a parasite density of $108 \mathrm{P} / \mu \mathrm{l}$ (SD: 32.6; [40; 200]), confirming a persistent infection two days after birth.

3.3. Clinical Features of Congenital Malaria. 60\% (21/35) of newborns had at least one general sign after delivery. $51.43 \%(18 / 35)$ of newborns had a temperature above $37.5^{\circ} \mathrm{C} .42 .86 \%(15 / 35)$ of newborns had an Apgar at eight [8]. 20\% (7/35) of newborns had a Blantyre score of 4 . $51.43 \%(18 / 35)$ of newborns did not have suction reflex. $2.86 \%$ of newborns $(1 / 35)$ had cyanosis, and $8.57 \%(3 / 35)$ had pallor. $11.43 \%(4 / 35)$ had seizures. $20 \%(7 / 35)$ of newborns had coma. $8.57 \%(3 / 35)$ had jaundice, and $45.71 \%$ $(16 / 35)$ had low birth weight.

$97.14 \%(34 / 35)$ of neonates were treated with artemether-lumefantrin in oral suspension for three days and $2.86 \%(1 / 35)$ with injectable artesunate. All the thick blood smear of the treatment control was negative. There was no relapse after treatment. The average duration of treatment was three days. All newborns were cured, and no deaths were recorded.

3.4. Biological Characteristics of Congenital Malaria. The mean parasite density of neonates with congenital malaria disease was $108 \mathrm{P} / \mu \mathrm{l}$ (SD: 32.6; [40; 200]). The average temperature was $37.6^{\circ} \mathrm{C}(\mathrm{DS}=0.5,[36.5,39.2])$. The mean heart rate was 155 beats per minute $(S D=10.69 ;[135 ; 180])$. The mean respiration rate was 49 per minute $(\mathrm{SD}=8.2$; [34; $66])$. The mean haemoglobin level was $14.8 \mathrm{~g} / \mathrm{dl}(\mathrm{SD}=2.6$, $[7.7,19.9])$. The mean haematocrit was $40.7 \%(\mathrm{SD}=6.9$, $[21 ; 54]$ ). The platelet average was 259.4 (DS $=93205$, $[88,000,459,000])$. The average number of white blood cells was $14057(\mathrm{SD}=6955,[4300,38,000])$. The mean glucose level was $0.79 \mathrm{~g} / \mathrm{L}(\mathrm{SD}=0.54,[0.26,1.87])$. The average of C-Reactive Protein (CRP) was 8.7 (DS = 6; [5; 24]).

\subsection{Determinants of Transmission of Congenital Malaria}

3.5.1. Prenatal Consultations. $95.18 \%$ of pregnant women had prenatal consultations. Twenty-six point ten percent (65/249) of the mothers had three antenatal visits during pregnancy. $24.10 \%(60 / 249)$ completed four prenatal consultations, and $4.82 \%(4 / 35)$ did not attend prenatal consultations. Prenatal consultation is not a protective factor against congenital malaria. $88.57 \%(31 / 35)$ of neonates from prenatal mothers have had congenital malaria compared to $11.43 \%$ (4/35) of nonattenders. There is no difference in congenital malaria between the groups of women who consult and those who do not consult.

3.5.2. Intermittent Preventive Treatment (IPT). Twenty-seven point thirty-one percent (68/249) of pregnant women received no dose of sulfadoxine-pyrimethamine (SP) during pregnancy. $24.9 \%(62 / 249)$ received one and two IPT, respectively. $22.09 \%(55 / 249)$ received three intermittent preventive treatments. Newborns of mothers who do not take IPT are more exposed to congenital malaria. In fact, $63 \%(22 / 35)$ of newborns of mothers who did not take IPT made congenital malaria compared to $37 \%(13 / 35)$. Children of mothers who did three IPTs had less congenital malaria $(5.71 \%)$ than mothers who did two IPTs (28.57\%) and those who did not receive IPT (37.14\%).

3.5.3. Use of Insecticide-Treated Mosquito Nets. Sixty-eight point sixty-seven percent (171/249) of women did not sleep under a long-lasting insecticide-treated bed net. Newborns of women who use LLINs have had less congenital malaria than women who do not use it. There is a statistically significant difference in congenital malaria between the two groups $(p=0.04)$. Long-lasting insecticidal mosquito net is a protective factor against congenital malaria.

3.5.4. Notion of Fever or Malaria during Pregnancy. Twentyfour point one percent (60/249) of women had fever during pregnancy. Newborns of women who have had a fever during pregnancy are half likely to be exposed to congenital malaria. 
TABLE 1: Characteristics of the population.

\begin{tabular}{|c|c|c|c|c|}
\hline Characteristics & Mothers & Newborns & $\mathrm{SD}$ & IC95 \\
\hline Number included & 249 & 249 & - & - \\
\hline Mean âge & 27 years & - & 6.2 & {$[12 ; 42]$} \\
\hline Sex ration & - & 0.9 & - & - \\
\hline Average weight (g) & - & 2754 & 588 & {$[1050 ; 4600]$} \\
\hline Average size $(\mathrm{cm})$ & - & 47.23 & 3.31 & {$[34 ; 54]$} \\
\hline Congénital malaria (\%) & - & 26.51 & - & - \\
\hline Parasite density (P/ùl) & - & 101 & 47.3 & {$[80 ; 320]$} \\
\hline Congenital malaria disease (\%) & - & 14.06 & - & - \\
\hline Parasite density (P/ùl) & - & 108 & 32.6 & {$[40 ; 200]$} \\
\hline
\end{tabular}

TABLe 2: Analysis of factors associated with congenital malaria.

\begin{tabular}{|c|c|c|c|c|}
\hline Variable & Number & $\begin{array}{c}\text { Congenital Malaria }(\%) \\
(12) 00(17) 00(26) 00\end{array}$ & $\begin{array}{l}\text { Rapport of } \\
\text { prevalence }\end{array}$ & $\begin{array}{c}p \\
\text { value }\end{array}$ \\
\hline \multicolumn{5}{|c|}{ Prenatal consultation } \\
\hline Yes & 31 & $88.57 \%$ & \multirow{2}{*}{7} & \multirow{2}{*}{ NS } \\
\hline No & 4 & $11.43 \%$ & & \\
\hline \multicolumn{5}{|c|}{ Usage of long-lasting insecticide-treated net } \\
\hline Yes & 12 & $34.29 \%$ & \multirow{2}{*}{1.17} & \multirow{2}{*}{0.04} \\
\hline No & 23 & $65.71 \%$ & & \\
\hline \multicolumn{5}{|c|}{$\begin{array}{l}\text { Intermittent preventive treatment with sulfadoxine- } \\
\text { pyrimethamine }\end{array}$} \\
\hline Yes & 13 & $37 \%$ & \multirow{6}{*}{1.9} & \multirow{6}{*}{ NS } \\
\hline No & 22 & $63 \%$ & & \\
\hline 0 IPT & 13 & $37.14 \%$ & & \\
\hline $1 \mathrm{IPT}$ & 10 & $28.57 \%$ & & \\
\hline 2 IPT & 10 & $28.57 \%$ & & \\
\hline 3 IPT & 2 & $5.71 \%$ & & \\
\hline \multicolumn{5}{|c|}{ Notion of fever and malaria treatment during pregnancy } \\
\hline Yes & 8 & $23 \%$ & \multirow{2}{*}{2.1} & \multirow{2}{*}{0.5} \\
\hline No & 27 & $77 \%$ & & \\
\hline
\end{tabular}

3.6. Analysis of Factors Associated with Congenital Malaria. Table 2 summarizes the analysis of all the factors that could be associated with congenital malaria, in particular prenatal consultation, use of insecticide-treated nets (IDNs), intermittent preventive treatment (IPT), and notion of malaria or fever during pregnancy.

\section{Discussion}

This cross-sectional and prospective study, conducted from $1^{\text {th }}$ of June to $30^{\text {th }}$ of November 2017 at the Neonatology Department of Issaka Gazobi Maternity in Niamey, describes the epidemiological, clinical, and biological characteristics of congenital malaria, analyses the factors of its transmission, and finally proposes preventative measures.

The study was conducted on 249 pairs of mothers and newborns. The prevalence of congenital malaria infection, objectified by a positive thick blood slide, was $26.51 \%$ $(66 / 249)$ with an average parasite density of $101 \mathrm{P} / \mu \mathrm{l}$ (SD:
47.3; [80; 320]). Congenital malaria infection is common in sub-Saharan Africa [14]. Its prevalence varies from $10.8 \%$ to $54.2 \%$ depending on the intensity of transmission [8], the collection period, and the biological confirmation method. The first study conducted in Niger in 2000 gave a prevalence of $13.3 \%$ [15]. It was $19 \%$ in Togo with a parasite density ranging from 360 to $870 \mathrm{P} / \mu \mathrm{L}$ [19], 10\% in Burkina [20], 2.2\% in Ghana [21], 0.64\% in Brazzaville [22], 0.03\% in Mali [23], and 0\% in Burundi [24]. Menendez and Mayor explain this wide variation in the prevalence of congenital malaria infection by: (I) the difference in the definition of congenital malaria, (II) the level of maternal immunity, (III) the type of blood examined (peripheral blood or the umbilical cord blood of the newborn), (IV) the expertise of microscopists, (V) the method of biological confirmation (microscopy, rapid diagnostic test, or chain polymerization reaction), and finally, (VII) the intensity of malaria transmission from the site [25]. In utero transmission of Plasmodium from mother to child by the placenta is accidental because the foetuses are normally protected by the maternal antibodies, foetal haemoglobin, and colostrum that the newborn receives [4].

The prevalence of congenital malaria disease is mostly low compared to that of congenital malaria infection [20]. On the other hand, the parasite density is most often high. It was $14.06 \%(35 / 249)$ with a parasite density of $108 \mathrm{P} / \mu \mathrm{l}$ (SD: 32.6, [40; 200] in Niger, a prevalence of $24.4 \%$ in Burkina Faso [2], 1.7\% in Togo with a parasite density ranging from 700 to $3000 \mathrm{P} / \mu \mathrm{L}$ [19], and $1.05 \%$ in Senegal [26].

The incriminated plasmodium species are most often Plasmodium falciparum. All infections were Plasmodium falciparum at the Issaka Gazobi Maternity in Niamey, as in Ghana [21]. Plasmodium vivax predominates (92.50\%) in Tanzania [12], and finally, P. falciparum and P. ovale were found in Mali [23].

Clinical manifestations of congenital malaria are most often not specific [11]. The main signs are fever, anaemia, and splenomegaly [8]. Jaundice, vomiting, and diarrhoea are also reported [12]. Biologically, it is characterized by parasitaemia [27] and thrombocytopenia [13]. In this series, hyperthermia, loss of sucking reflex, convulsion, coma, and pallor, and especially low birth weight are the most prominent signs. In fact, $45.71 \%$ of newborns had a low birth weight. The prognosis of congenital malaria is most often 
favorable. The treatment instituted was an artemisinin-based combination therapy suspension, especially artemetherlumefantrin. Prior to 2005, quinine was prescribed. However, since the AQUAMAT [28] and SEAQUAMAT [29] studies, artemether and artesunate have been prescribed [8]. No deaths were recorded in this study compared with $25 \%$ in Togo [19].

Only the nonuse of long-lasting insecticidal nets was significantly associated with congenital malaria $(p=0.04)$. Newborns of mothers with fever during pregnancy are at least twice at risk of congenital malaria. Fever is a bad prognostic factor for congenital malaria. Intermittent preventive treatment tends to show a protective effect against congenital malaria: Only thirty-seven percent of newborns in IPT mothers have had congenital malaria compared to $63 \%$ of those who do not. The effect of IPT also increases with the dose. Only $5.71 \%$ of newborns of mothers who did three IPTs had congenital malaria compared to $28 \%$ of those who had one dose of IPT.

Several recommendations are made to diagnose and treat congenital malaria. Francisca et al. believes that in malariaendemic areas, any newborn with a sepsis chart should be investigated for possible congenital malaria. This recommendation will be very strong if the mother has not correctly taken antimalaria prophylaxis [30]. Other alternatives are also possible such as microscopic examination of placentas. For the prevention of congenital malaria, in the stable malaria transmission zone, the use of long-lasting insecticide-treated bed nets [31] and intermittent preventive treatment for pregnant women with sulfadoxine-pyrimethamine are the mains recommendations of WHO [25, 32].

The main limitation of this study is the lack of a serological assessment to confirm the exposure and use of PCR to highlight submicroscopic infections.

\section{Conclusion}

The frequency of congenital malaria infection is very high in Niamey where transmission is permanent with a strong recrudescence in the rainy season. On the other hand, the prevalence of congenital malaria disease is lower. The main risk factor is the lack of use of mosquito nets. Intermittent preventive treatment and distribution of long-lasting insecticide-treated mosquito nets should be provided to all pregnant women to prevent congenital malaria.

\section{Data Availability}

The excel file data used to support the findings of this study are available from the corresponding author upon request.

\section{Conflicts of Interest}

The authors declare that there is no conflict of interest.

\section{Authors' Contributions}

TI led the study, made, and read the slides. ZM and KM led the fieldwork and consulted newborns; MML participated in the design of the study and the writing of the manuscript. IA, DM, and SA participated in writing the manuscript. IML designed the study, analyzed the data, and drafted the manuscript.

\section{Acknowledgments}

We thank Dr. Jean Testa, Scientific Director of CERMES for reading and editing the manuscript. Consumables and reagents of laboratory (cotton, alcohol, slides, dyes, etc.) were provided by the Parasitology Unit of CERMES. The blood exams were carried out by the laboratory of biology of the Issaka Gazobi Maternity.

\section{References}

[1] P. N. Mbanzulu and K. Kapepela, Programme WHOMA. Paludisme congénital clinique à la maternité des cliniques universitaires du Mont Amba à Kinshasa, Zaire, World Health Organization, Geneva, Switzerland, 1988, http://apps.who .int/iris/handle/10665/59171.

[2] K. Nagalo, F. Dao, P. Minodier et al., "Le paludisme congénital maladie à Plasmodium falciparum: aspects épidémiologiques, cliniques, biologiques, thérapeutiques et pronostiques à Ouagadougou, Burkina Faso," Pan African Medical Journal, vol. 18, 2014.

[3] R. W. Steketee, B. L. Nahlen, M. E. Parise, and C. Menendez, "The burden of malaria in pregnancy in malaria-endemic areas," The American Journal of Tropical Medicine and Hygiene, vol. 64, 1 Supplement, pp. 28-35, 2001.

[4] V. Moya-Alvarez, R. Abellana, and M. Cot, "Pregnancy-associated malaria and malaria in infants: an old problem with present consequences," Malaria Journal, vol. 13, no. 1, p. 271, 2014.

[5] J. Testa, J. Awodabon, N. Lagarde, T. Olivier, and J. Delmont, "Plasmodial indices and malarial placentopathy in 299 parturients in Central Africa," Medecine tropicale: revue du Corps de sante colonial, vol. 50, no. 1, pp. 85-90, 1990.

[6] I. A. McGregor, M. E. Wilson, and W. Z. Billewicz, "Malaria infection of the placenta in The Gambia, West Africa; its incidence and relationship to stillbirth, birthweight and placental weight," Transactions of the Royal Society of Tropical Medicine and Hygiene, vol. 77, no. 2, pp. 232-244, 1983.

[7] P. Bouvier, B. Kouriba, O. Doumbo et al., "Seasonality, malaria, and impact of prophylaxis in a West African Village I. Effect on anemia in pregnancy," The American Journal of Tropical Medicine and Hygiene, vol. 56, no. 4, pp. 378-383, 1997.

[8] C. J. Uneke, "Congenital malaria: an overview," Tanzania Journal of Health Research, vol. 13, no. 3, 2011.

[9] H. L. Guyatt and R. W. Snow, "Malaria in pregnancy as an indirect cause of infant mortality in sub-Saharan Africa," Transactions of the Royal Society of Tropical Medicine and Hygiene, vol. 95, no. 6, pp. 569-576, 2001.

[10] L. E. Fitri, N. E. Jahja, I. R. Huwae, M. B. Nara, and N. BerensRiha, "Congenital malaria in newborns selected for low birthweight, anemia, and other possible symptoms in Maumere, Indonesia," The Korean Journal of Parasitology, vol. 52, no. 6, pp. 639-644, 2014. 
[11] S. A. Shah, Z. Ahmed, M. A. Lodhi, and N. A. Malik, "Congenital malaria," Journal of Ayub Medical College, Abbottabad, vol. 27, no. 3, pp. 721-722, 2015.

[12] Z. Tao, Q. Fang, X. Liu et al., "Congenital Malaria in China," PLoS Neglected Tropical Diseases, vol. 8, no. 3, article e2622, 2014.

[13] S. Gülaşı and N. Özdener, "Congenital malaria: importance of diagnosis and treatment in pregnancy," The Turkish Journal of Pediatrics, vol. 58, no. 2, pp. 195-199, 2016.

[14] P. R. Fischer, "Congenital malaria: an African survey," Clinical Pediatrics, vol. 36, no. 7, pp. 411-413, 1997.

[15] A. Djibo and A. Cénac, "Paludisme congénital. Etudes parasitologique et sérologique à Niamey (Niger)," Cahiers d'études et de recherches francophones/Santé, vol. 10, no. 3, pp. 183-187, 2000.

[16] A. Souman and G. Tankar, "Contribuation à l'étude du paludisme congenital," Sciences et Médecine Revue cames, vol. 4, pp. 24-28, 2006.

[17] H. Soumaila, M. Idrissa, M. Akgobeto et al., "Multiple mechanisms of resistance to pyrethroids in Anopheles gambiae s. 1 populations in Niger," Médecine et Maladies Infectieuses, vol. 47, no. 6, pp. 415-423, 2017.

[18] OMS, Diagnostic microscopique du paludisme: manuel d'assurance qualité - Ver. 2, World Health Organization, 2020, [cited 2020 Aug 24], http://www.who.int/malaria/publications/atoz/ 9789241549394/fr/.

[19] B. Balaka, A. D. Agbere, P. Bonkoungou, K. Kessie, K. Assimadi, and K. Agbo, "Paludisme congénital-maladie à Plasmodium falciparum chez le nouveau-né à risque infectieux," Archives de Pédiatrie, vol. 7, no. 3, pp. 243-248, 2000.

[20] H. M. Natama, D. F. Ouedraogo, H. Sorgho et al., "Diagnosing congenital malaria in a high-transmission setting: clinical relevance and usefulness of P. falciparum HRP2-based testing," Scientific Reports, vol. 7, no. 1, p. 2080, 2017.

[21] C. C. Enweronu-Laryea, G. O. Adjei, B. Mensah, N. Duah, and N. B. Quashie, "Prevalence of congenital malaria in high-risk Ghanaian newborns: a cross-sectional study," Malaria Journal, vol. 12, no. 1, 2013.

[22] J. A. Mbongo, G. E. Bowassa, C. K. Gombet, and L. H. Iloki, "Paludisme Congénital au Centre Hospitalier et Universitaire de Brazzaville : une Étude Épidémiologique de 90 Cas," Health Sciences and Diseases, vol. 16, no. 1, 2015.

[23] F. Dicko-Traoré, M. Sylla, A. A. Djimdé et al., "Le paludisme congénital et néonatal en Afrique subsaharienne, un évènement rare?," Journal de Pédiatrie et de Puériculture, vol. 24, no. 2, pp. 57-61, 2011.

[24] J. Stassijns, W. van den Boogaard, P. Pannus, A. Nkunzimana, and A. Rosanas-Urgell, "Prevalence and diagnostics of congenital malaria in rural Burundi, a cross-sectional study," Malar J, vol. 15, no. 1, p. 443, 2016.

[25] C. Menendez and A. Mayor, "Congenital malaria: The least known consequence of malaria in pregnancy," Seminars in Fetal and Neonatal Medicine, vol. 12, no. 3, pp. 207-213, 2007.

[26] F. N. Diouf, "Prévalence du paludisme congénital infestation a plasmodium falciparum au centre Hospitalier Régional de Ziguinchor/Sénégal," Revue Africaine et Malgache de Recherche Scientifique/Sciences de la Santé, vol. 3, no. 1, 2015.

[27] S. M. Bugvi and N. Ahmed, "Congenital malaria: a rare entity," Journal of the College of Physicians and Surgeons-Pakistan, vol. 25, no. 11, pp. 841-842, 2015.

[28] A. M. Dondorp, C. I. Fanello, I. C. E. Hendriksen et al., “Artesunate versus quinine in the treatment of severe falciparum malaria in African children (AQUAMAT): an open-label, randomised trial," The Lancet, vol. 376, no. 9753, pp. 1647$1657,2010$.

[29] W. Nick, "Artesunate versus quinine for treatment of severe falciparum malaria: a randomised trial," The Lancet, vol. 366, no. 9487 , pp. 717-725, 2005.

[30] M. Francisca, C. David, K. Hélène, and O. M. Thérèse, "Paludisme Congénital : Difficultés Diagnostiques chez un Nouveau-né au Centre Hospitalier Universitaire de Yaounde, Cameroun," Health Sciences and Diseases, vol. 14, no. 3, 2013.

[31] WHO, Achieving and Maintaining Universal Coverage with Long-Lasting Insecticidal Nets for Malaria Control, WHO, 2019, [cited 2019 Jan 2], http://www.who.int/malaria/ publications/atoz/who_recommendation_coverage_llin/en/.

[32] OMS, Traitement préventif intermittent du paludisme (TPI) pour les femmes enceintes, WHO, 2019, [cited 2019 Jan 2], http://www.who.int/malaria/areas/preventive_therapies/ pregnancy/fr/. 